1 This is the accepted version of the following article: Morales J (2020) Eggshell Biliverdin as an

2 Antioxidant Maternal Effect: Biliverdin as an Antioxidant Resource in Oviparous Animals.

3 BioEssays 42: 2000010, which has been published in final form at:

4 https://onlinelibrary.wiley.com/doi/abs/10.1002/bies.202000010. This article may be used for

5 non-commercial purposes in accordance with the Wiley Self-Archiving Policy

6 [http://www.wileyauthors.com/self-archiving].

7

8

9

\title{
Eggshell biliverdin as an antioxidant maternal effect
}

\author{
Judith Morales
}

12

National Museum of Natural Sciences, Spanish National Research Council (CSIC), c/ José

Gutiérrez Abascal 2, 28006 Madrid, Spain. Correspondence: jmorales@mncn.csic.es; Tel.: +34

914111328 (ext. 981341).

Subtitle: Eggshell biliverdin as an antioxidant resource in oviparous animals

Article type: Hypotheses

Author contribution: JM conceived the research and wrote the paper.

Data availability statement: No data was used. 
In this essay, I propose the hypothesis that biliverdin pigment plays an antioxidant role in the avian eggshell. Due to its ability to scavenge free radical species and to reduce mutation, biliverdin potentially counteracts the oxidative action of pathogens that penetrate the eggshell and/or protects the shell membrane from oxidation, thus promoting the proven antioxidant and antimicrobial capacities of the shell membrane itself. Additionally, biliverdin may be able to inhibit viral replication in the eggshell due to its ascribed antiviral properties. Moreover, previous results in other taxa leave open the question of whether biliverdin can be absorbed by the embryo from the eggshell and play a role in embryogenesis. These mechanisms of antioxidant action of eggshell biliverdin remain totally unexplored in birds and in other oviparous animals. I develop the main assumptions and predictions of the antioxidant hypothesis, and propose directions for future research.

Keywords: antioxidants, biliverdin pigmentation, eggshell colouration, eggshell membrane, maternal effects, oxidative stress 


\section{2 \\ 1. Introduction}

The colour of avian eggs has long attracted evolutionary ecologists. In the nineteenth century, renowned naturalists such as Darwin, Wallace and Hewitson already observed its variation across species and suggested that it was related to nesting habits. ${ }^{[1]}$ Since then, the function of avian eggshell colouration has remained a major topic in the field (for some reviews and discussions in the past two decades, $\left.\operatorname{se}^{[1-6]}\right)$. Recently, attention has also been directed to understanding the evolutionary origin of eggshell colouration. Indeed, the two main pigments present in avian eggs, protoporphyrin (responsible for brown hues) and biliverdin IX $\alpha$ (for blue and green hues), have even been studied and identified in dinosaur eggshells. ${ }^{[7]}$

Adaptive explanations proposed for variation in eggshell pigmentation can be included in two main, non-mutually exclusive sets of hypotheses. The first one focuses on eggshell colour as an external trait that can be visually perceived by a diverse array of observers. It can favour camouflage from predators ${ }^{[8]}$ and avian brood parasites, ${ }^{[9]}$ or the opposite, it can increase conspicuousness to signal egg unpalatability to predators. ${ }^{[10]}$ In species with biparental care, shell colour has been proposed as a post-mating signal of female quality to encourage greater paternal investment ("sexual signalling hypothesis"), ${ }^{[3]}$ or to force it ("blackmail hypothesis"). ${ }^{[1]]}$ The second set of hypotheses is based on the direct action of protoporphyrin and biliverdin, whose chemical properties can adaptively modulate the structure and physiology of the shell and thus influence embryonic survival. Specifically, protoporphyrin has been suggested to enhance eggshell strength ${ }^{[4,12]}$ and to promote a photoactive antimicrobial defence. ${ }^{[13]}$ Biliverdin, through interactions with the light environment, has been proposed to accelerate embryonic development and to favour the light-dependent repair of DNA lesions caused by specific UV radiation. ${ }^{[14]}$ This interaction would only apply to open-nesting species; however, the evidence in these species is mixed. ${ }^{[15,}$

${ }^{16]}$ On the other hand, it has been reported that both pigments reduce near-infrared absorbance and solar overheating. ${ }^{[17]}$ Again, these actions would be associated mainly with 
open-nesting species exposed to high levels of harmful radiation. In contrast, in a comparative analysis performed at a global scale, Wisocki and co-authors concluded that brown eggshell colouration is more frequent in open-nesting species living in cold and humid climates. ${ }^{[6]}$ Moreover, they found that darker eggs heated more rapidly than lighter ones when exposed to solar radiation, supporting a thermoregulatory role for both eggshell pigments (biliverdin and protoporphyrin) combined. Nonetheless, although the analysis yielded an impressive dataset, in which the eggshell colour of more than 600 species belonging to most avian orders was measured, the interspecific patterns of eggshell blue-green chroma were not explained by the environmental factors studied. ${ }^{[6]}$

An often overlooked property of biliverdin that has the potential to directly promote embryo survival is its antioxidant capacity. Traditionally, biliverdin and its derivative in humans, bilirubin, have been considered as toxic, but this is mainly evident when the concentration of bile pigments is unusually high. ${ }^{[18]}$ Nowadays, biliverdin's ability to scavenge free radical species and reduce mutation is also clearly recognized ${ }^{[19-22]}$ (in birds, see ${ }^{[23]}$ ). Certainly, the sexual signalling hypothesis of blue-green eggshell colouration is grounded in biliverdin's antioxidant properties: females that lay more colourful blue-green eggs signal their low oxidative status to males. ${ }^{[3]}$ In the present essay, however, I argue that these antioxidant properties also have the potential to directly promote embryo survival. This "antioxidant hypothesis" is motivated by the facts that i) biliverdin's molecular structure (a tetrapyrrole with an extended system of conjugated double bonds and reactive hydrogen atoms) is responsible for its antioxidant properties ${ }^{[21]}$ and ii) the biliverdin molecule in the eggshell is exactly the same as in other organismal tissues in which it plays an antioxidant role. ${ }^{[21,24]}$ According to the antioxidant hypothesis, biliverdin counteracts the oxidative action of pathogens that penetrate the eggshell and/or protects the shell membrane from oxidation, thus promoting the proven antioxidant and antimicrobial capacities of the eggshell membrane itself. ${ }^{[25]}$ Biliverdin might also directly inhibit viral replication in the eggshell, due to its ascribed 
antiviral properties. ${ }^{[26]}$ Moreover, as previously speculated, ${ }^{[27]}$ it is not implausible to hypothesize that the avian embryo gradually absorbs biliverdin traces from the eggshell, as it does with calcium. Biliverdin IX $\alpha$, the same molecule used by female birds to pigment their eggs, is also present in the oocyte, egg and embryonic cytoplasm of the amphibian Xenopus laevis, and plays a crucial role in dorsal axis development. ${ }^{[28]}$ Although amphibian eggs differ significantly from avian eggs, mainly because they lack a shell and pigments mostly accumulate in the yolk, the study in X. laevis leaves open to question a direct function of eggshell biliverdin during avian embryonic development. Similarly, in insects, CV-bilin, a biliverdin isomer present in eggs has been proposed to function in cellular regulation. ${ }^{[29]}$

To my knowledge, these mechanisms of antioxidant action of eggshell biliverdin (Table 1) remain totally unexplored. In the following two sections, I develop the assumptions and predictions of the antioxidant hypothesis of eggshell biliverdin, the previous observations that support them, and possible ways to test the hypothesis.

\section{Conceptual framework of the antioxidant hypothesis}

\subsection{Assumptions: inherent antioxidant capacity and high permeability of biliverdin}

There are two main assumptions underlying the antioxidant hypothesis of eggshell biliverdin.

The first one is that the biliverdin molecule possesses the ability to scavenge free radical species and to reduce mutation, which, as mentioned above, has been consistently demonstrated (e.g., ${ }^{[19-22]}$ ). By reducing oxidative damage and mitigating infections, eggshell biliverdin potentially protects the integrity of the thin organic membrane that envelops the albumen and connects the true shell with the egg contents (Figure 1). Thus, the second assumption is that biliverdin extends deep into the calcified layers of the eggshell and can come in direct contact with the eggshell membrane. Indeed, small amounts of the pigment (less than 0.01 absorbance/g) have been detected in the innermost calcified eggshell layers in various bird species ${ }^{[30,31]}$ (see also ${ }^{[7]}$ in dinosaur eggs), which can be visually perceived in some 
cases (Figure 2a). This high permeability also implies that the molecule surrounds eggshell pores and cracks, gateways for microbes. Intriguingly, pore density has been positively associated with biliverdin-based eggshell pigmentation in a passerine species at a wide geographic scale. ${ }^{[32]}$

Additionally, in the case that the embryo absorbs eggshell biliverdin as a resource during development, a third assumption should be that the pigment permeates the shell membrane itself. This assumption is supported by three arguments. First, the shell membrane penetrates the basal calcified layer, ${ }^{[33,34]}$ also called the cone or mammillary layer, which can be permeated by biliverdin (Figure 1). Second, protoporphyrin, which is less permeable than biliverdin, ${ }^{[35]}$ has been identified in the shell membrane of various bird species. ${ }^{[36]}$ Third, biliverdin shows high affinity to carrier proteins that are abundant both in the shell membrane and in the albumen, ${ }^{[33,37]}$ like albumin, globulin and vitellogenin, ${ }^{[33,38]}$ the major yolk precursor protein associated with embryonic development. Biliverdin also interacts with cysteine-rich proteins, ${ }^{[39]}$ which are abundant in the shell membrane, and forms soluble complexes with egg mammillary layer. ${ }^{[40]}$

If biliverdin is absorbed by the embryo during development, one question to address is why do females not allocate it directly inside the egg. One possible reason is that biliverdin assimilation is similar to that of eggshell calcium, which is gradually absorbed within fine physiological limits and whose excess inside the egg can be highly toxic for the embryo. ${ }^{[41]}$ If true, I would expect biliverdin traces to be more easily detected in the shell membrane at advanced stages of embryo development, coinciding with a higher exposure to oxidative stress. ${ }^{[42]}$ Suggestive of this, I have observed that the shell membrane of the blue-footed booby

143 (Sula nebouxi) partially has a greenish tint at hatching (Figure 2a, arrow); nonetheless, the pigment composition of this membrane needs to be confirmed analytically. In this context, one may ask how biliverdin could be transported inside the egg. On the one hand, eggshell 
membrane proteins represent a potential mechanism, given the high affinity of biliverdin for them. On the other hand, calcium is implicated in biliverdin synthesis in the shell gland and its transport to uterine fluid, ${ }^{[43]}$ suggesting the possibility that the embryo gradually absorbs biliverdin together with calcium. The latter mechanism would depend, first, on the possibility that biliverdin-calcium complexes can be formed, and then on the relative amount of eggshell protoporphyrin pigment, which shows high affinity for calcium. ${ }^{[24]}$

\subsection{Predictions: highly conserved but only abundant under high oxidative stress}

If biliverdin plays a beneficial antioxidant role during development, the main prediction should be that most bird species allocate it in the eggshell. However, there are many birds that do not lay blue-green eggshells, ${ }^{[1,6]}$ at least apparently. One possibility is that biliverdin traces are indeed present in the eggshells of most species and that they are enough to elicit an antioxidant effect. In fact, it has been claimed that unpigmented eggs are rare in nature and that most white eggs contain pigment traces, ${ }^{[50]}$ but this needs to be demonstrated.

As most studies on biliverdin focus on signalling mechanisms or eggshell colour as a proxy for pigment concentration, the prevalence of biliverdin traces is unknown in most cases. However, apparently unpigmented eggs of some species have been shown to contain biliverdin (e.g., the eggs of the black-footed and the Humboldt's penguin, the Mandarin duck and the wood pigeon, respectively, Spheniscus demersus, S. humboldti, Aix galerlculata and Columba palumbus; ${ }^{[51]}$ see also ${ }^{[52]}$ in white Leghorns, Gallus gallus domesticus), and brown eggs can contain it in very high amounts. ${ }^{[16,51]}$ In all the previous cases, the presence of biliverdin would go unnoticed if only blue-green eggshell colouration is relied on as a measure. Note also that the eggs of many marine and raptor species are blue only when fresh and become white or grey almost immediately after laying (see Figure $2 \mathrm{~b})^{[53]}$. Nonetheless, it is possible to observe that the inner shell layers of such eggs at hatching contain high amounts of biliverdin (Figure 2a). Colour fading of the outermost eggshell layers also occurs in 
songbirds. ${ }^{[54]}$ Thus, the antioxidant hypothesis predicts, in the first place, that most species contain biliverdin traces in the inner eggshell layers, regardless of eggshell external appearance. Only in species in which biliverdin-based pigmentation has evolved as a signal (directed at predators, brood parasites or males) or as a photo-protective agent should it be present in high amounts in the outermost shell layers as well.

$$
\text { Although the physiology of closely related species is not expected to differ to the }
$$
extent that biliverdin is either allocated or not in the eggshell, it is plausible that the associated costs for females limit the amount allocated. In fact, the allocation of biliverdin to the eggshell has been shown to decrease antioxidant levels in the defence system of laying females, ${ }^{[55]} \mathrm{a}$ cost that is alleviated when the females are supplemented with carotenoids and antioxidant vitamins prior to egg laying. ${ }^{[27,46,56]}$ Thus, a second prediction of the hypothesis is that large amounts of the pigment are allocated to the eggshell only in those species in which the antioxidant benefits for the embryo compensate the oxidative costs for females. Due to the ability of biliverdin to scavenge free radical species, thus reducing DNA damage, its role should be more prominent when embryos are particularly exposed to oxidation during development. First, life-history strategies characterized by rapid embryonic growth and small body mass and brain size have been consistently linked to increased oxidative damage across divergent taxonomic groups. ${ }^{[57-59]}$ Second, high amounts of biliverdin in the inner shell layers are predicted to be more relevant in the case of plastic increases in growth rate, for example, catch-up growth strategies in late-hatching animals, which can lead to higher oxidative damage. ${ }^{[60]}$ Third, biliverdin traces are predicted to be more easily detected in species or populations that are more exposed to external sources of oxidative stress, such as pathogens and pollutants. Previous results seem to support these predictions. For instance, biliverdin eggshell colouration has been reported to be more frequent in smaller species ${ }^{[61,62,15]}$ (but see ${ }^{[1]}$ ) and in passerine lineages with shorter incubation periods. ${ }^{[15]}$ Intraspecific studies have also reported that biliverdin-based pigmentation is associated with higher haemolytic bacterial 
loads in the eggshell, ${ }^{[47]}$ and is related to environmental contamination. ${ }^{[63,64]}$ The latter relationships could merely be due to changes in haem biosynthesis following high exposure to pathogens or contaminants. ${ }^{[65]}$ However, it is also possible that eggshell pigmentation is in part adaptively upregulated to protect the embryo against external sources of oxidation. Finally, a third prediction of the antioxidant hypothesis is that the shell membrane shows stronger antioxidant and antimicrobial properties when biliverdin is present, especially in the inner shell layers. Furthermore, by enhancing the properties of the shell membrane, which is the last barrier between the true shell and the albumen, biliverdin would also be protecting the egg contents. Then, we may likewise predict that the yolk and the albumen show greater antioxidant capacity when eggshell biliverdin is present in the inner shell layers. In agreement with the latter prediction, in various bird species, biliverdin-based pigmentation reflects the antioxidant quality of egg contents ${ }^{[44-46]}$ (but see $\left.{ }^{[47]}\right)$, including yolk vitamin $E$ and carotenoids, both of which protect against oxidation. Moreover, in poultry species, blueshelled egg yolks possess higher radical scavenging activity than white-shelled egg yolks, ${ }^{[48]}$ one of the reasons commercial blue eggs are more valued in Korea ${ }^{[48]}$. Although none of the above studies demonstrated a direct physiological function of biliverdin in the shell, they have revealed that its allocation is narrowly linked to the antioxidant quality of bird mothers ${ }^{[99]}$ and their eggs. ${ }^{[44-46]}$

\section{Testing the hypothesis}

The antioxidant hypothesis assumes that biliverdin is in direct contact with the shell membrane and, if absorbed by the embryo, permeates the shell membrane itself (Table 1). Further studies on the antioxidant role of eggshell biliverdin should thus aim to detect biliverdin traces/concentration directly in the innermost calcified layers and in the shell membrane. This has been achieved by means of layer-by-layer dissolution methods combined with spectrophotometric measurements of the supernatant. ${ }^{\left[{ }^{[0]}\right.}$ The shell membrane can be 
easily detached from the calcified layers and be analysed separately. Additionally, the use of Raman spectroscopy has been successfully used in eggshell fragments ${ }^{[7,66]}$ and can map pigments across vertical egg sections. ${ }^{[7]}$ Given that the external eggshell colour do not accurately reflect the concentration of pigments in the inner layers (Fig. 2b), ${ }^{[67]}$ spectrophotometric methods should focus on the inner eggshell surface. This may limit the scope for studying, for instance, specimens from Museum egg collections. However, in natural populations, broken eggshells can be easily collected at hatching. Actually, if biliverdin is gradually absorbed by the embryo, I would expect that it is more easily detected in the shell membrane at later stages of embryo development.

The antioxidant capacity of the shell membrane and the egg contents (yolk and albumen) needs to be compared between species that lay eggs pigmented with biliverdin and those that do not, ${ }^{[48]}$ and also among species that allocate biliverdin in different amounts. The use of a battery of tests (i.e., assessment of total antioxidant capacity, as well as radical scavenging activity, yolk lipid peroxidation and the amount of specific antioxidants like lysozyme, vitamin E, carotenoids, superoxide dismutase and glutathione peroxidase) would contribute to a better characterization of the antioxidant capacity of different egg components. ${ }^{[48]}$ Intriguingly, the eggshells of certain bird species also contain biliverdin reductase (Hanley, D., personal communication), which is the enzyme that catalyses the reduction of biliverdin to bilirubin in other vertebrates. ${ }^{[20]}$ Future studies could also explore the occurrence of this molecule in the different shell layers and test whether it increases the antioxidant potential of biliverdin.

However, to reveal causal relationships between the presence of biliverdin in the eggshell and the antioxidant potential of the shell membrane/egg components, experimental manipulation of biliverdin levels at laying is needed. Experiments of this kind have been performed by exogenous injection of the pigment into the shell gland of ducks, Anas platyrhynchos, ${ }^{[31]}$ although this approach would entail more difficulty in smaller species. A 
complementary manipulation would be to induce an external oxidative challenge during embryo development (e.g., elevated oxygen concentration during incubation) ${ }^{[42]}$ and analyse the resulting relationship between eggshell biliverdin concentration and the oxidative status of the shell membrane and egg components. In addition, it would be extremely interesting to assess biliverdin concentration in the plasma of hatchlings in response to the above experimental approaches. Increased pigment concentration in plasma after experimental enhancement of the pigment in the eggshell would support absorption by the embryo during development.

The hypothesis also predicts that eggshell biliverdin should be more important when the embryo is more exposed to oxidative stress (Table 1). Inter- and intra-specific analyses could test this prediction by exploring the occurrence of eggshell biliverdin traces in relation to species/populations growth strategies and to exposure to pathogens and contaminants. Interspecific studies should control for potential selective forces known to favour biliverdin-based pigmentation (at least, in the outermost eggshell layers), like nesting habits, male parental care, predation risk and light exposure.$^{[1,3,8,68]}$ As mentioned in the preceding section, the relationship with microbes and contaminants could merely reveal changes in haem biosynthesis pathway following high exposure to these external oxidative agents or could also be due to an adaptive upregulation of biliverdin synthesis. Experimental manipulation of the exposure to pathogens and contaminants could help to distinguish between these two possibilities. An adaptive response would be supported if: i) eggshell biliverdin is upregulated after the experimental challenge and ii) is accompanied by a decrease in oxidative stress.

\section{Conclusions and outlook}

In this essay, I propose the hypothesis that biliverdin pigment functions as an antioxidant in the eggshell, and potentially in the shell membrane and the embryo. Due to the ability of biliverdin to scavenge free radical species, thus reducing DNA damage, the hypothesis predicts 
it to act as an antioxidant in most bird species, but its role should be more prominent when embryos are particularly exposed to oxidation. Future research should focus on studying the presence of biliverdin traces in the inner eggshell layers and in the link of these traces with embryonic exposure to oxidative damage, mediated, for instance, by fast growth strategies, exposure to pollution or microbial infections. Experimental studies manipulating eggshell biliverdin to explore its effect on the eggshell membrane and on embryonic development are also promising lines of research. The antioxidant hypothesis of biliverdin in the eggshell (and beyond it) is compatible with other explanations proposed for blue-green eggshell pigmentation, for example, the idea that biliverdin can accelerate embryonic development due to a photo-active effect, predicted to be more important in species with faster growth rates. ${ }^{[14,}$ that vary according to both the life history of a species and the environment, ${ }^{[6]}$ thus making it difficult to elucidate its adaptive function. This essay does not pretend to offer a unique explanation for the observable patterns of blue-green eggshell colouration. Rather, it aims to reopen the debate about these unexplained patterns with a different perspective, one based on the antioxidant potential of biliverdin in the eggshell, an ancient biomolecule that was already present in dinosaur eggshells, well before the modern bird radiation. ${ }^{[7]}$ This and other hypotheses on the role of biliverdin during embryogenesis need to be further explored in birds and in other taxa.

ACKNOWLEDGEMENTS: I thank Juan Moreno and Manuel Jiménez-Tenorio for insightful discussions on the ideas presented here. I am also thankful to Daniel Hanley for invaluable comments and to Melinda Modrell for English correction of a previous version of this paper. This work was supported by a Ramón y Cajal by the Ministerio de Economía, Industria y Competitividad MINECO (CGL2016-79390-P) and the European Regional Development Fund (FEDER). 
304 The author declares no conflict of interest.

305

306

REFERENCES

307

[1] R. M. Kilner, Biol. Rev. 2006, 81, 383.

308

[2] T.J. Underwood, S.G. Sealy, in Avian Incubation, Behaviour, Environment and Evolution (Ed:

309

D.C. Deeming) Oxford University Press, Oxford 2002, pp. 280-298.

310

[3] J. Moreno, J.L. Osorno, Ecol. Lett. 2003, 6, 803.

311

[4] A.G. Gosler, J.P. Higham, S.J. Reynolds, Ecol. Lett. 2005, 8, 1105.

312

[5] P. Cassey, Curr. Biol. 2009, 19, 1083.

313

[6] P.A. Wisocki, P. Kennelly, I. Rojas-Rivera, P. Cassey, D. Hanley, Nature Ecol. Evol. 2020, 4, 148.

[7] J. Wiemann, T-R. Yang, M.A. Norell, Nature 2018, 563, 555.

[8] M. C. Stoddard, K.L.A. Marshall, R.M. Kilner, Avian Biol. Res. 2011, 4, 196.

317

[9] N.B. Davies, M.De L. Brooke, J. Anim. Ecol. 1989, 58, 207.

318

[10] H.B. Cott, Nature 1948, 161, 8.

319

[11] D. Hanley, S. Doucet, D.C. Dearborn, The Auk 2010, 127, 453.

320

[12] S.E. Solomon, Egg and Eggshell Quality, lowa State University Press, Ames, IA 1997.

321

[13] S.I. Ishikawa, K. Suzuki, E. Fukuda, K. Arihara, Y. Yamamoto, T. Mukai et al., FEBS Lett. $2010,584,770$.

[14] G. Maurer, S.J. Portugal, P. Cassey, J. Avian Biol. 2011, 42, 494.

[15] J.J. Soler, J. Moreno, J.M. Avilés, A.P. Møller, Evolution 2005, 59, 636. Soc. 2012, 106, 657. 
[18] S.F. Asad, S. Singh, A. Ahmad, N.U. Khan, S.M. Hadi, Chem. Biol. Interact. 2001, 137, 59.

[19] R. Stocker, Proc. Natl. Acad. Sci. USA 1987, 84, 5918.

[20] A.F. McDonagh, Nat. Struct. Biol. 2001, 8, 198.

[21] H. Kaur, M.N. Hughes, C.J. Green, P. Naughton, R. Foresti, R. Motterlini, FEBS Lett. 2003, $543,113$.

[22] A.C. Bulmer, K. Ried, J.T. Blanchfield, K.-H. Wagner, Mut. Res. 2008, 658, 28.

[23] J.L. Baylor, M.W. Butler, J. Exp. Biol. 2019, 222, 1.

[24] G.Y. Kennedy, H.G. Vevers, Comp. Biochem. Physiol. 1973, 44B, 11.

[25] Y. Shi, J. Kovacs-Nolan, B. Jiang, R. Tsao, Y. Mine, J. Funct. Foods 2014, 10, 35.

[26] Z. Zhu, A.T. Wilson, B.A. Luxon, K.E. Brown, M.M. Mathahs, S. Bandyopadhyay, et al., Hepatology 2010, 52, 1897.

[27] J. Morales, A. Velando, R. Torres, Behav. Ecol. Sociobiol. 2011, 65, 197.

[28] K.H. Falchuk, J.M. Contin, T.S. Dziedzic, Z.L. Feng, T.C. French, G.J. Heffron, et al., Proc. Natl Acad. Sci. USA 2002, 99, 251.

[29] H. Kayser, V. Wray, M. Nimtz, FEBS J. 2014, 281, 2366.

[30] X.-T. Wang, X.-M. Deng, C.-J. Zhao, J.-Y. Li, G.-Y. Xu, L.-S. Lian, C.-X. Wu, Poult. Sci. 2007, $86,2236$.

[31] H.C. Liu, M.C. Hsiao, Y.H. Hu, S.R. Lee, W.T.K. Cheng, Asian-Aust. J. Anim. Sci. 2010, 23, 162.

[32] J. Morales, S. Ruuskanen, T. Laaksonen, T. Eeva, R. Mateo, E. Belskii, E. et al., J. Avian Biol. 2013, 44, 111.

[33] J. Gautron, Y. Nys, in: Bioactive egg compounds, Springer, Berlin 2007, pp. 103-108.

[34] S.G. Tullet, Comp. Biochem. Physiol. 1984, 78A, 5.

[35] L. R. Milgrom, M.J. Warren, in The Colours of Life: An Introduction to the Chemistry of Porphyrins and Related Compounds (Ed: L.R. Milgrom), Oxford University Press, Oxford 1997, pp. 1-175. 
[36] M.R. Lang, J.W. Wells, World. Poultry Sci. J. 1967, 43, 238.

[37] V.K. Kodali, S.A. Gannon, S. Paramasivam, S. Raje, T. Polenova, C. Thorpe, PLoS One 2011,

$$
6,1 .
$$

[38] G.V. Marinetti, J.T. Bagnara, Science 1983, 219, 985.

[39] J. Wiemann, T-R. Yang, P.N. Sander, M. Schneider, M. Engeser, S. Kath-Schorr, C.E. Müller, P.M. Sander, PeerJ 2017, 5, e3706.

[40] M.T. Hincke, J. Gautron, M. Panhéleux, J.M. Garcia-Ruiz, M.D. McKee, Y. Nys, Mater. Biol. $2000,19,443$.

[41] B.M. Freeman, M.A. Vince, in: Development of the avian embryo. Springer, Dordrecht 1974, pp. 237-248.

[42] H. Watson, P. Salmón, C. Isaksson, J. Exp. Biol. 2018, 221, 1.

[43] Z. Wang, G. Meng, Y. Bai, R. Liu, Y. Du, L. Su, BMC genomics 2017, 18, 725.

[44] R. Hargitai, C. Moskát, M. Bán, D. Gil, I. López-Rull, E. Solymos, J. Avian Biol. 2010, 41, 177.

[45] C. Navarro, T. Pérez-Contreras, J.M. Avilés, K.J. McGraw, J.J. Soler, J. Avian Biol. 2011, 42, 538.

[46] M.W. Butler, K.J. McGraw, Funct. Ecol. 2013, 27, 1176.

[47] H. Hoi, A. Darolová, J. Krištofík, M. Poláček, J. Majtán, M. Zeman, et al., Ethol. Ecol. Evol. 2019, 31, 249.

[48] J. Sujiwo, D. Kim, J.-Y. Yoon, H. Kim, J.-S. Kim, S.-K. Lee, et al., Korean J. Food Sci. An. 2017, $37,181$.

[49] D. Hanley, G. Heiber, D.C. Dearborn, The Condor 2008, 110, 767.

[50] M.D. Shawkey, L. D’Alba, Nature 2019, 570, E43.

[51] G.Y. Kennedy, H.G. Vevers, Comp. Biochem. Physiol. 1976, 55B, 117.

[52] T. Tamura, S. Fujii, J. Fac. Fish. Anim. Husb. 1967, 7, 35.

[53] J. Morales, R. Torres, A. Velando, Naturwissenschaften 2010, 97, 173.

[54] J. Moreno, E. Lobato, J. Morales, Ornis Fenn 2011, 88, 51. 
380 [55] J. Morales, A. Velando, J. Moreno, Behav. Ecol. Sociobiol. 2008, 63, 227.

381 [56] R. Hargitai, N. Boross, Z. Nyiri, Z. Eke, Behav. Ecol. Sociobiol. 2016, 70, 2093.

382 [57] A.P. Martin, S. Palumbi, Proc. Natl. Acad. Sci. USA 1993, 90, 4087.

383 [58] I. Galván, J. Erritzøe, F. Karadaş, A.P. Møller, J. Comp. Physiol. B 2012, 182, 947.

384 [59] P. Monaghan, N.B. Metcalfe, R. Torres, Ecol. Lett. 2009, 12, 75.

385 [60] N. Metcalfe, C. Alonso-Álvarez, Funct. Ecol. 2010, 24, 984.

386 [61] D. Lack, Ibis 1958, 100, 145.

387 [62] M. Schönwetter, Handbuch der Oologie, Akademie Verlag, 1960 -1992.

388 [63] A. Jagannath, R.F. Shore, L.A. Walker, P.N. Ferns, A.G. Gosler, J. Apppl. Ecol. 2008, 45, 133.

389 [64] D. Hanley, S. Doucet, J. Appl. Ecol. 2012, 49, 1055.

390 [65] R. Mateo, G. Castells, A.J. Green, C. Godoy, C. Cristòfolb, J. Chromat. 2004, 810, 305.

391 [66] D.B. Thomas, M.E. Hauber, D. Hanley, G.I.N. Waterhouse, S. Fraser, K.C. Gordon, J. Exp.

392 Biol. 2015, 218, 2670.

393 [67] D. Hanley, T. Grim, P. Cassey, M.E. Hauber, Biol. Lett. 2015, 11, 20150087.

394 [68] G. Maurer, S.J. Portugal, M.E. Hauber, I. Mikšík, D.G.D. Russell, P. Cassey, Funct. Ecol. 2015, 29, 209. 


\begin{tabular}{|c|c|c|}
\hline Proposed specific action & Assumptions/predictions & Ways to test assumptions/predictions \\
\hline \multirow[t]{3}{*}{$\begin{array}{l}\text { BLV protects the shell } \\
\text { membrane against oxidation }\end{array}$} & $\begin{array}{l}\text { - BLV traces present in most bird species } \\
\text { - High permeability of BLV through the inner } \\
\text { calcified layers }\end{array}$ & $\begin{array}{l}\text { - Apply analytical methods (e.g., layer-by-layer } \\
\text { dissolution; Raman spectroscopy) to different shell } \\
\text { layers }{ }^{[7,30,66]}\end{array}$ \\
\hline & $\begin{array}{l}\text { - BLV more abundant when the embryo is more } \\
\text { exposed to oxidation }\end{array}$ & $\begin{array}{l}\text { - Explore BLV traces/concentration in species } \\
\text { /populations that differ in growth strategies and } \\
\text { exposure to pathogens and contaminants }\end{array}$ \\
\hline & $\begin{array}{l}\text { - Higher antioxidant capacity of the shell } \\
\text { membrane/egg contents when BLV is } \\
\text { present/more abundant }\end{array}$ & $\begin{array}{l}\text { - Experimental manipulation of BLV at laying or of } \\
\text { oxidative stress during embryo development; }{ }^{[31,42]} \\
\text { investigate differences in shell membrane/egg } \\
\text { antioxidant capacity in relation to BVL }{ }^{[48]}\end{array}$ \\
\hline $\begin{array}{l}\text { Biliverdin traces are absorbed } \\
\text { by the embryo as an } \\
\text { antioxidant resource }\end{array}$ & $\begin{array}{l}\text { Additional to the above: } \\
\text { - } \quad \text { BLV permeates the shell membrane } \\
\text { BLV more abundant at later stages of embryo } \\
\text { development }\end{array}$ & $\begin{array}{l}\text { - Analytical methods (see above) })^{[7,30,66]} \text { applied to } \\
\text { inner shell layers at different stages of development; } \\
\text { assessment of biliverdin levels in the plasma of } \\
\text { hatchlings after BLV manipulation at laying }\end{array}$ \\
\hline
\end{tabular}


401 Figure 1. Structure of a typical avian eggshell showing both the previously reported extent of 402 biliverdin in the innermost calcified layers ${ }^{[30,31]}$ and the potential reach in the shell membrane proposed in this essay. Reprinted from World's Poultry Science Journal, M.R. Lang \& J.W. Wells,

404 A Review of eggshell pigmentation, page 240 , Copyright (1987), and minimally modified with permission from Taylor \& Francis Ltd (http://www.tandfonline.com; license: 4792060147918) and from Elsevier

407 (https://www.sciencedirect.com/science/article/abs/pii/0300962984900835; license number: 4784730822058), publisher of the original figure in Comparative Biochemistry and Physiology Part A: Physiology, 78 (1), S.G. Tullet, The porosity of avian eggshells, page 5, Copyright (1984)

411 Figure 2. a) Inner surface of a blue-footed booby (Sula nebouxii) eggshell on the hatching day.

412 Note the intense biliverdin-based blue-green colouration. The arrow points to remaining shell 413 membrane that has a greenish tint; b) external surface of a blue-footed booby eggshell close to 414 hatching. Credit: J. Morales. 


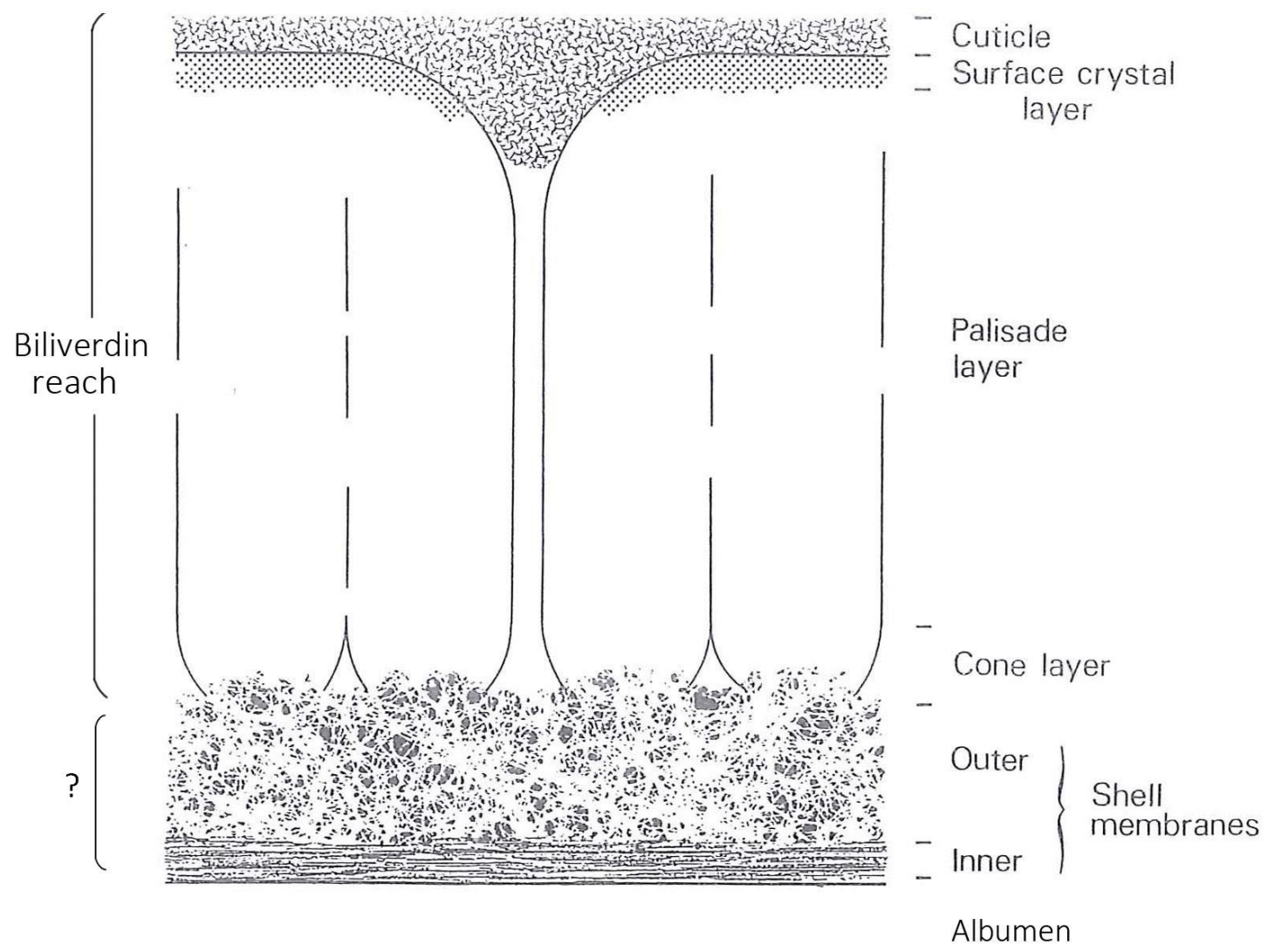

415

$416 \quad$ Fig. 1 


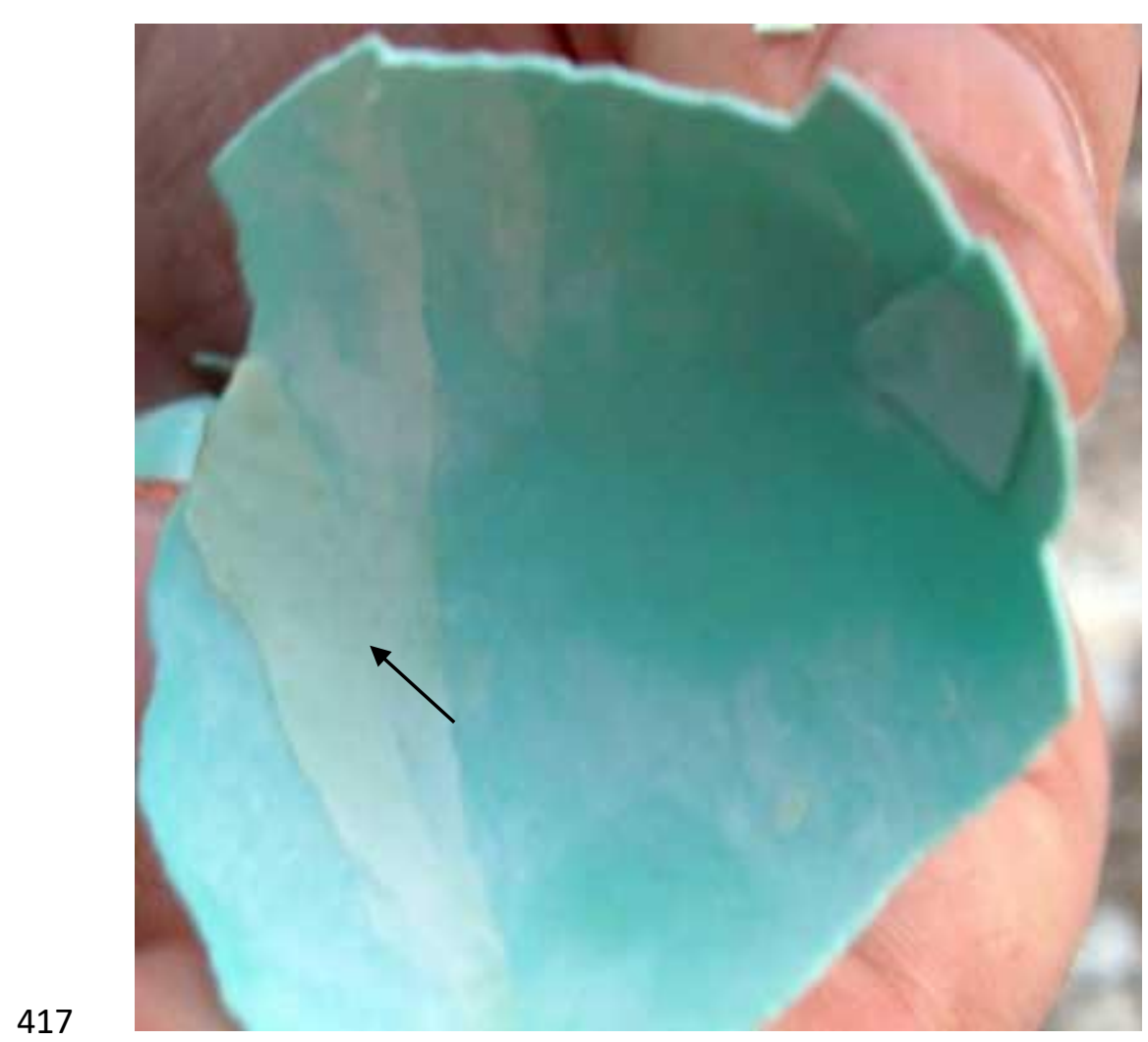

$418 \quad$ Fig. 2a

419 


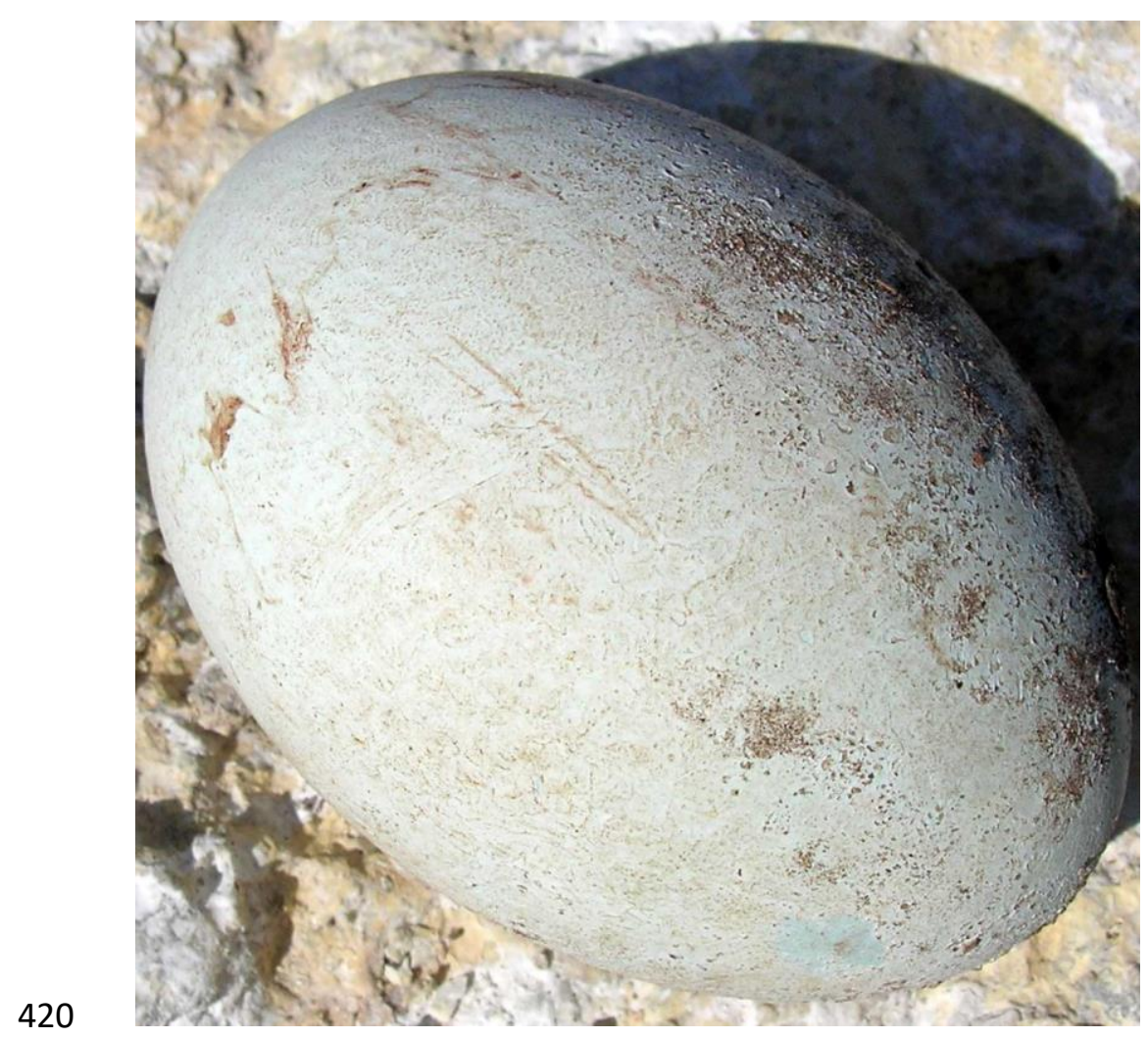

$421 \quad$ Fig. 2b

422 